\title{
(6) OPEN ACCESS \\ Global prevalence of visual impairment associated with myopic macular degeneration and temporal trends from 2000 through 2050: systematic review, meta-analysis and modelling
}

\author{
Timothy R Fricke, ${ }^{1}$ Monica Jong, ${ }^{1,2}$ Kovin S Naidoo, ${ }^{1,2,3}$ Padmaja Sankaridurg, ${ }^{1,2}$ \\ Thomas J Naduvilath, ${ }^{1}$ Suit May Ho, ${ }^{1}$ Tien Yin Wong, ${ }^{4}$ Serge Resnikoff ${ }^{1,2}$
}

- Additional material is published online only. To view please visit the journal online (http://dx.doi.org/10.1136/ bjophthalmol-2017-311266).

${ }^{1}$ Brien Holden Vision Institute, Sydney, Australia

${ }^{2} \mathrm{School}$ of Optometry and Vision Science, University of New South Wales, Sydney, Australia

${ }^{3}$ African Vision Research Institute, University of KwaZuluNatal, Durban, South Africa ${ }^{4}$ Singapore Eye Research Institute, Singapore National Eye Center, Duke-NUS Medical School, National University of Singapore, Singapore, Singapore

\section{Correspondence to} Professor Kovin S Naidoo, Brien Holden Vision Institute, University of New South Wales, Kensington, NSW 2052,Australia; k.naidoo@ brienholdenvision.org

Received 31 August 2017 Accepted 27 March 2018 Published Online First 26 April 2018
Check for updates

To cite: Fricke TR, Jong $\mathrm{M}$,

Naidoo KS, et al.

Br J Ophthalmol

2018:102:855-862.

\section{ABSTRACT}

Purpose We used systematic review and meta-analysis to identify and assimilate evidence quantifying blindness and visual impairment (VI) associated with myopic macular degeneration (MMD), then derived models to predict global patterns. The models were used to estimate the global prevalence of blindness and $\mathrm{VI}$ associated with MMD from 2000 to 2050.

Methods The systematic review identified 17 papers with prevalence data for MMD VI fitting our inclusion criteria. Data from six papers with age-specific data were scaled to relative age-dependent risk and meta-analysed at $\mathrm{VI}$ and blindness levels. We analysed variance in all MMD VI and blindness data as a proportion of high myopia against variables from the place and year of data collection, with a model based on health expenditure providing the best correlation. We used this model to estimate the prevalence and number of people with MMD VI in each country in each decade.

Results We included data from 17 studies comprising 137514 participants. We estimated 10.0 million people had VI from MMD in 2015 (prevalence 0.13\%, $95 \%$ Cl 5.5 to 23.7 million, $0.07 \%$ to $0.34 \%$ ), 3.3 million of whom were blind $(0.04 \%, 1.8$ to 7.8 million, $0.03 \%$ to $0.10 \%$ ). We estimate that by 2050, without changing current interventions, VI from MMD will grow to 55.7 million people $(0.57 \%$, 29.0 to 119.7 million, $0.33 \%$ to $1.11 \%$ ), 18.5 million of whom will be blind $(0.19 \%, 9.6$ to 39.7 million, $0.11 \%$ to $0.37 \%$ ).

Conclusion The burden of MMD blindness and VI will rise significantly without efforts to reduce the development and progression of myopia and improve the management of MMD.

\section{INTRODUCTION}

Uncorrected refractive error is the main cause of presenting visual impairment (VI) globally, and myopia is the most common refractive error. ${ }^{12}$ Even with refractive correction, those with myopia are at higher risk of a range of conditions, including cataract, glaucoma, retinal pathologies such as tears and detachment, and myopic macular degeneration (MMD). ${ }^{3-5}$ MMD alone has been found to cause $12.1 \%$ of VI (approximately 200000 people) in Japan. $^{6}$
The prevalence of myopia and high myopia has been observed to be increasing globally. ${ }^{2}$ While this appears most dramatic in young East Asians, it is increasingly observed across other regions and age groups. ${ }^{2}$ Trends in lifestyle, education and demographics mean global myopia and high myopia prevalence is projected to continue rising. ${ }^{2}$ Estimates of the impact of this trend on the epidemiology of VI from MMD would inform planning for prevention and management of the problem. However, despite many prevalence studies on myopia, there are no estimates of the regional or global prevalence of VI caused by high myopia or MMD, or projected future changes.

This paper reviews population-based and blindness registry studies to estimate the global prevalence of $\mathrm{VI} /$ blindness associated with MMD, with modelling and projections to 2050. An expanded background and additional references can be found in online supplementary file 1 .

\section{METHODS}

\section{Studies, databases and data organisation}

We performed a systematic review of the prevalence of VI and blindness associated with MMD, summarised in figure 1 . Sixteen papers were included in our analysis of the prevalence of MMD VI/blindness. ${ }^{6-21}$ One additional paper was identified as having useful information on the age spread of myopia-related VI. ${ }^{4}$

The epidemiology of high myopia was taken from a systematic review and meta-analysis of the prevalence of myopia and high myopia. ${ }^{2}$ We searched for alternative models, but no comparable evidence was found.

Country-specific population data for each decade from 2000 through 2050, in 10-year age groups from 0 to $90+$, were drawn from the United Nations Department of Economic and Social Affairs. ${ }^{22}$ Countries were aggregated into the 21 Global Burden of Disease regions for data presentation. ${ }^{1}$

\section{Definitions}

High myopia was classified as spherical equivalent $\leq-5.00 \mathrm{D}$ in line with international agreement. ${ }^{23}$ MMD was defined as degenerative changes in the macula, including diffuse or patchy chorioretinal atrophy, lacquer cracks, choroidal neovascularisation and atrophy related to choroidal 


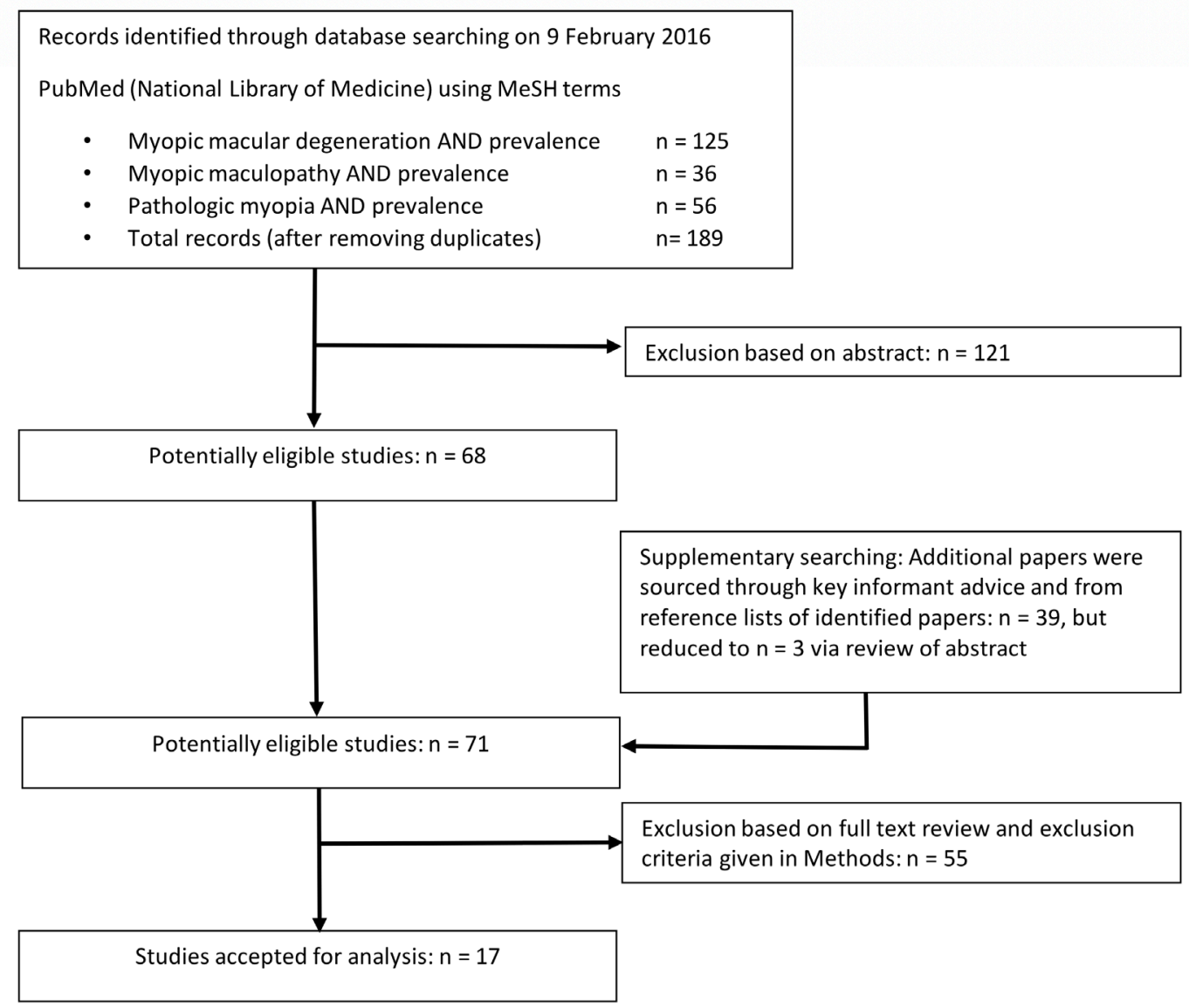

Figure 1 Flow diagram summarising the systematic search, using medical subject headings (MeSH) terms, and review process for identifying evidence on the prevalence of MMD VI and/or blindness. Our inclusion criteria were population-based or blindness registry studies quantifying prevalence, with sampling representative of whole communities. Exclusion criteria were unspecified or ambiguous definitions, not specifying the number of eligible participants, participation rate $<75 \%$, use of data duplicating other included studies or aggregating MMD VI with other conditions (eg, all macular conditions). MMD, myopic macular degeneration; VI, visual impairment.

neovascularisation, in the presence of higher levels of myopia. ${ }^{24}$ While MMD can occur in younger eyes with relatively low levels of myopia, it is more likely to occur in older, longer, more myopic eyes. ${ }^{45}$ Consequently, we translated the epidemiological evidence from population prevalence for a specific age group, year of data collection, country and urbanisation level into proportions of people with high myopia who have MMD VI using matched high myopia data. ${ }^{2}$ These proportions enabled more meaningful intercountry comparisons.

We used the WHO definitions of VI (visual acuity worse than $6 / 18$ ) and blindness (visual acuity worse than 3/60). ${ }^{25}$

\section{Meta-analysis and modelling}

Six papers provided detailed information on the relationship between age and prevalence of MMD VI and blindness. ${ }^{4810131418}$ Data from these six papers were scaled to relative age-dependent risk in 10-year age groups from 0 to $90+$. We used a standard meta-analysis tool (Comprehensive Meta-Analysis (V.3, Biostat, Englewood, New Jersey, USA)) to perform a weighted analysis of the relative risk in each age group. Regression analysis of the meta-analysis results against age found that exponential curves with $\mathrm{r}^{2}$ of 0.93 and 0.96 best described the relative risk of VI and blindness, respectively:

Relative risk of MMD VI $=0.00002 * \exp ^{(0.0756 *(\text { age }))}$, and Relative risk of MMD blindness $=0.00001 * \exp ^{(0.0796 *(a g e))}$.
We applied these age-specific relative risks of VI/blindness associated with MMD to data from all 16 papers in our main analysis, scaled to ensure that the overall VI/blindness associated with MMD remained equivalent to the primary data. We then combined age-specific MMD VI and blindness in 10-year age groups with the prevalence of high myopia data from the same urbanisation level of the same country, decade and age groups, ${ }^{2}$ to give the proportion of people with high myopia over 40 years of age who have MMD VI/blindness.

Analysis of the proportion data suggested there is some factor beyond the prevalence and age distribution of high myopia that affects not the prevalence of MMD itself but vision outcomes associated with MMD. Suspecting a factor related to country-level detection and management of myopia and consequent ability to prevent vision loss, we analysed the study time-specific and place-specific proportion of people with high myopia who have MMD VI or blindness against variables describing country-level development (full list and sources provided in online supplementary file 1 ). The following were the best correlations for the proportion of high myopes with MMD VI $\left(r^{2}=0.77\right)$ and blindness $\left(\mathrm{r}^{2}=0.85\right)$ :

$$
\begin{aligned}
& \mathrm{VI} \text { attributed to MMD }=0.1376 * \exp ^{(0.0008 *(H E))} \text {, and } \\
& \text { Blindness attributed to MMD }=0.0473 * \exp ^{(0.0009 *(H E))} \text {, }
\end{aligned}
$$

where $H E=$ health expenditure per capita in US\$. 
The equations are based on primary evidence covering $73 \%$ of the global population. We used the equations to calculate the proportion of high myopes who have VI and blindness associated with MMD, using each country's HE when that lay within the envelope of the primary evidence (US\$62-US\$2835 per capita), or using the closest limit of the envelope for countries with HE outside the envelope. ${ }^{26}$ We then combined the country-specific proportions with the prevalence of high myopia for each country, and the meta-analysed 0 to $90+$ age distribution of VI and blindness associated with MMD. The resulting country-specific prevalences for each 10 -year age group from 0 to $90+$ were combined with the population data to estimate the number of people with MMD VI and blindness. Crude and age-standardised all-ages prevalence of VI and blindness associated with MMD was calculated for each Global Burden of Disease region.

Projections for each decade to 2050 were based on the same model. HE was kept constant, so our projections across decades were based on change in high myopia, ${ }^{2}$ age demographics and population, ${ }^{22}$ and assumed a constant proportion of MMD VI to high myopia ratio.

\section{Confidence intervals}

In addition to the 95\% confidence limits calculated in the meta-analysis of relative risk between age groups and the 95\% confidence limits in high myopia estimates, uncertainty in future population projections was represented by the high and low variant projections from the United Nations. ${ }^{22}$

\section{RESULTS}

A description and summary of findings from the included studies are provided in the text and table S1 of online supplementary file 1.

Figure 2A,B shows our estimated age distribution of global MMD blindness in 2000 and 2050. Prevalence rises exponentially with age in both decades, but is scaled upwards in 2050 due to increasing high myopia and the spread of high myopia from younger groups in 2000 through all ages by 2050 .

Figure 3A,B illustrates our global estimates of people with MMD VI and blindness. In 2000, MMD VI was estimated to affect 4.2 million people, that is, $0.07 \%$ of the world population (95\% CI 2.3 to 9.8 million, $0.04 \%$ to $0.16 \%$ ), increasing

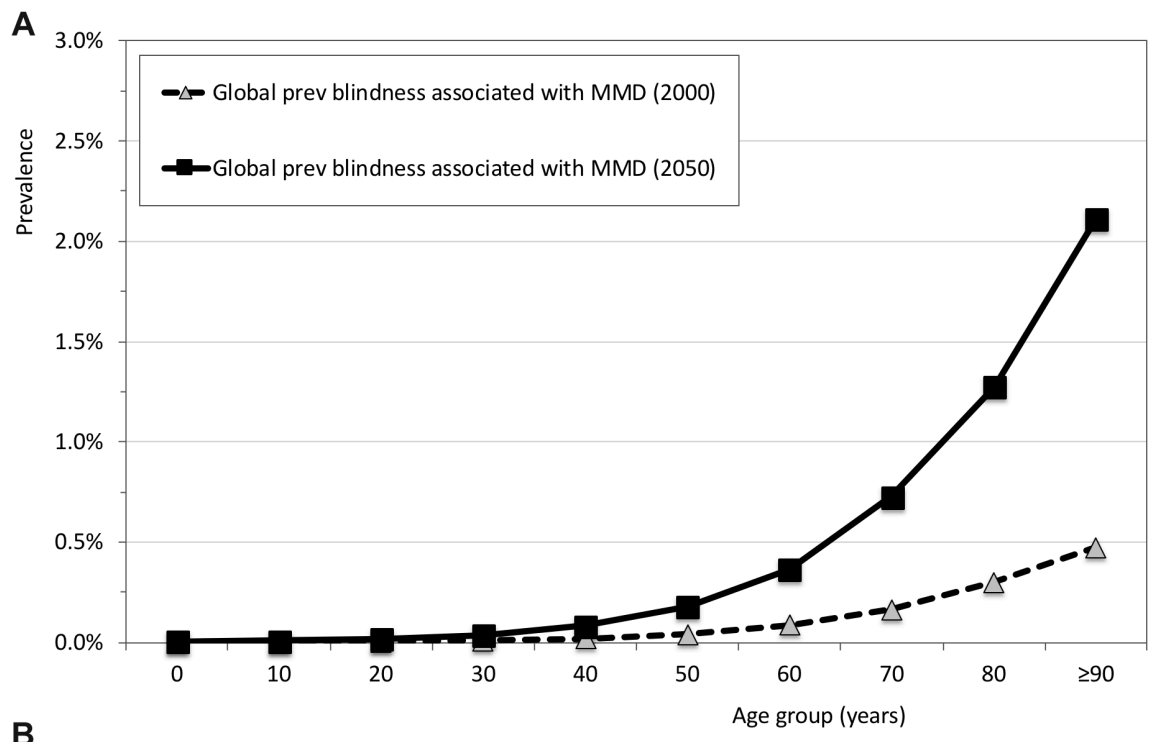

B

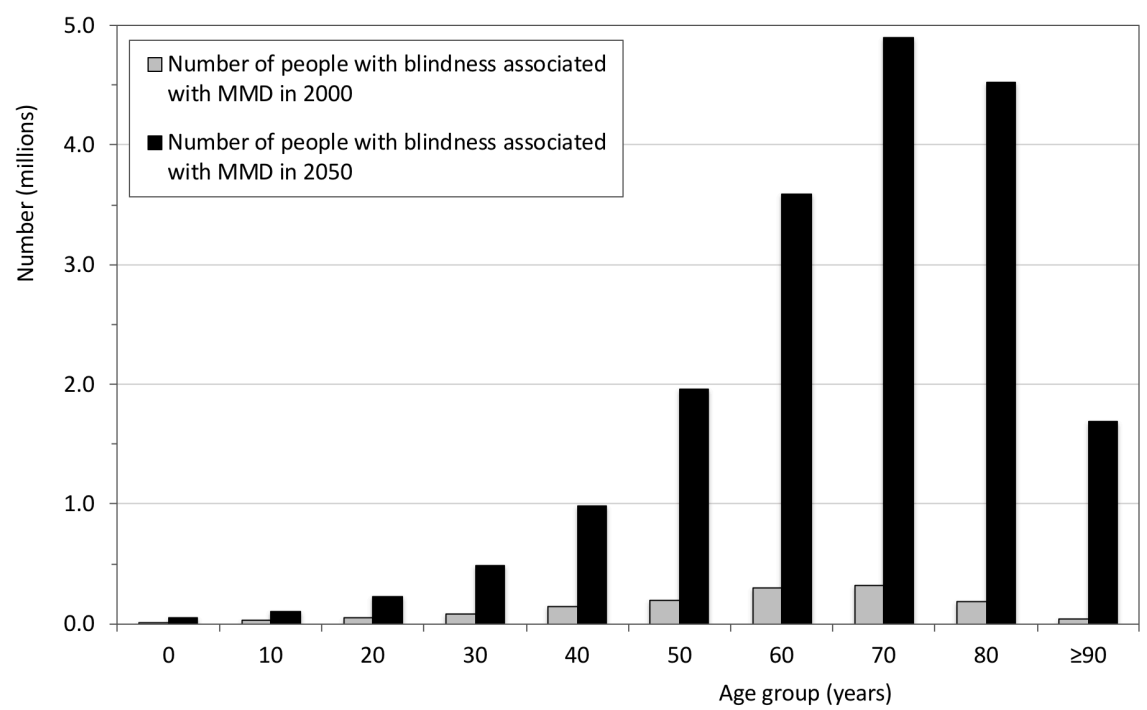

Figure 2 The age distribution of global blindness associated with myopic macular degeneration (MMD) in 2000 and 2050. Both age-specific global prevalence (top) and the number of people predicted to be blind (bottom) are shown. 


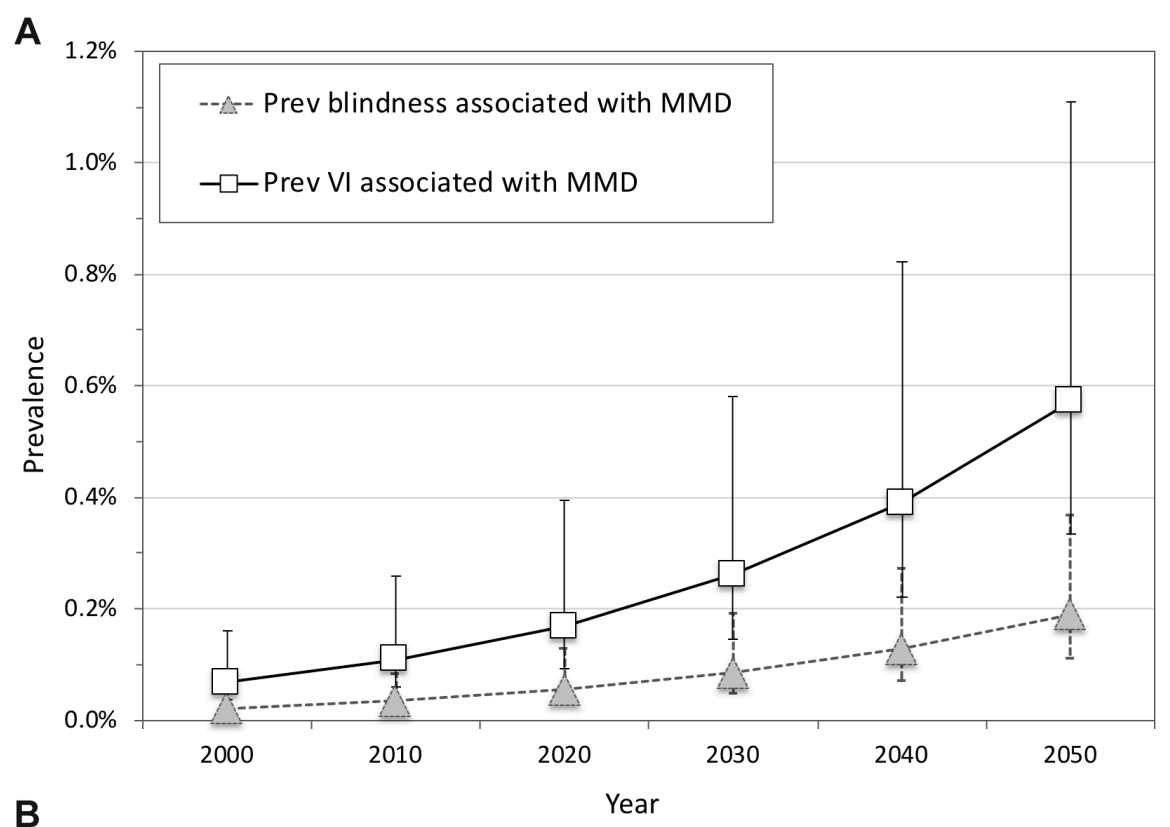

B

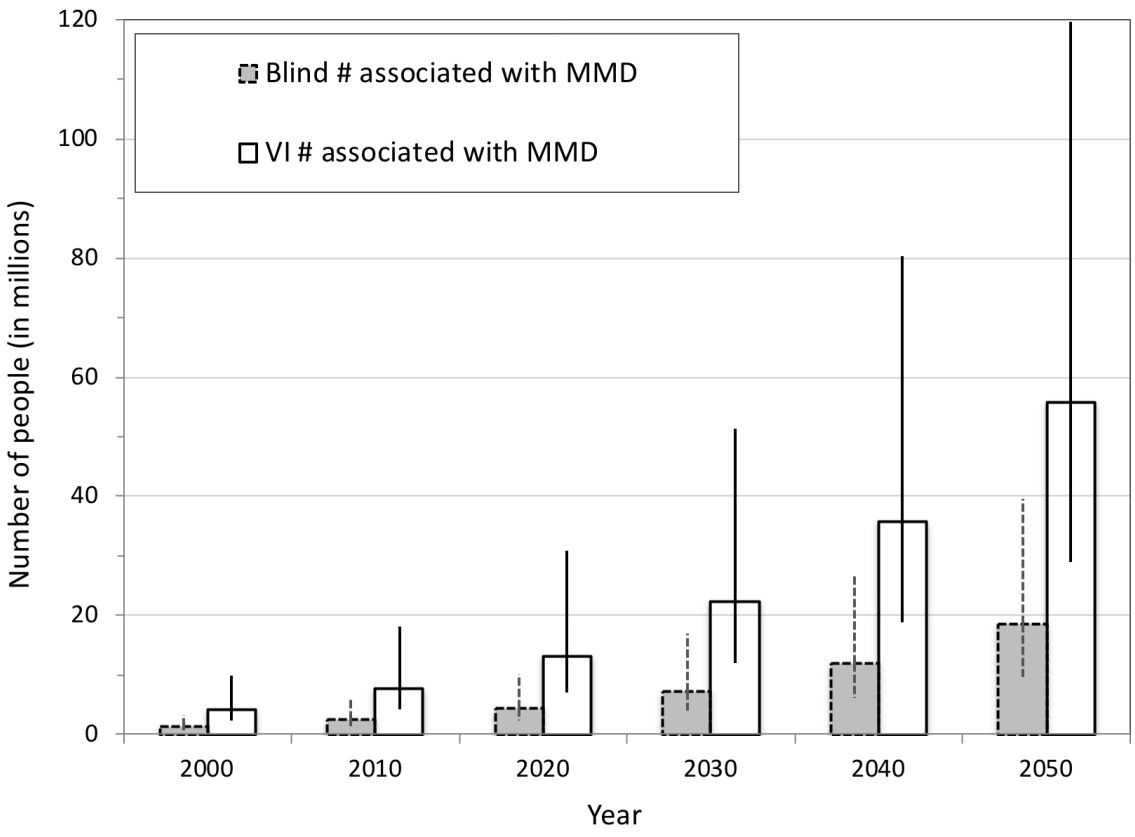

Figure 3 Predicted prevalence of, and number of people with, blindness and visual impairment (VI) associated with myopic macular degeneration (MMD) for each decade in 2000-2050. The top graph shows the crude all-ages prevalence of blindness (triangles and dashed line) and VI (squares with solid line) associated with MMD. The bottom graph shows the number of people with blindness (striped columns) and VI (unfilled columns) associated with MMD. Error bars represent $95 \% \mathrm{Cls}$.

to 55.7 million in 2050 , that is, $0.57 \%$ (29.0 to 119.7 million, $0.33 \%$ to $1.11 \%$ ). MMD blindness in 2000 was estimated to affect 1.3 million people, that is, $0.02 \%$ (0.8 to 3.2 million, $0.01 \%$ to $0.05 \%)$, increasing to 18.5 million in 2050 , that is, $0.19 \%$ (9.6 to 39.7 million, $0.11 \%$ to $0.37 \%$ ).

Table 1 shows the regional differences predicted by our model. In 2000, the all-ages prevalence of MMD VI ranged from $0.011 \%$ in Oceania (crude, or 0.019\% age-standardised) to $0.025 \%$ (crude, or $0.045 \%$ age-standardised) in Sub-Saharan Africa, to $0.097 \%$ (crude, or $0.111 \%$ age-standardised) in Asia (averaged across Central, East, South and South-East Asian regions). All regions were predicted to have an increase in prevalence of VI associated with MMD through to 2050, with Sub-Saharan Africa increasing to $0.179 \%$ (crude, or
$0.459 \%$ age-standardised) and Asia increasing to $0.960 \%$ (crude, or $0.871 \%$ age- standardised).

Additional details are available in online supplementary file 1.

\section{DISCUSSION}

Our evidence-based model predicts significant increases in VI and blindness associated with MMD, with consequent quality of life and socioeconomic impact, and therefore implications for planning comprehensive eye care services globally. Methods to reduce the development of myopia, progression to high myopia and management of MMD are all warranted to reduce expected burden. 
Table 1 The crude and age-standardised all-ages prevalence of visual impairment $(\mathrm{VI})$ and blindness associated with myopic macular degeneration (MMD) estimated for each Global Burden of Disease region between 2000 and 2050

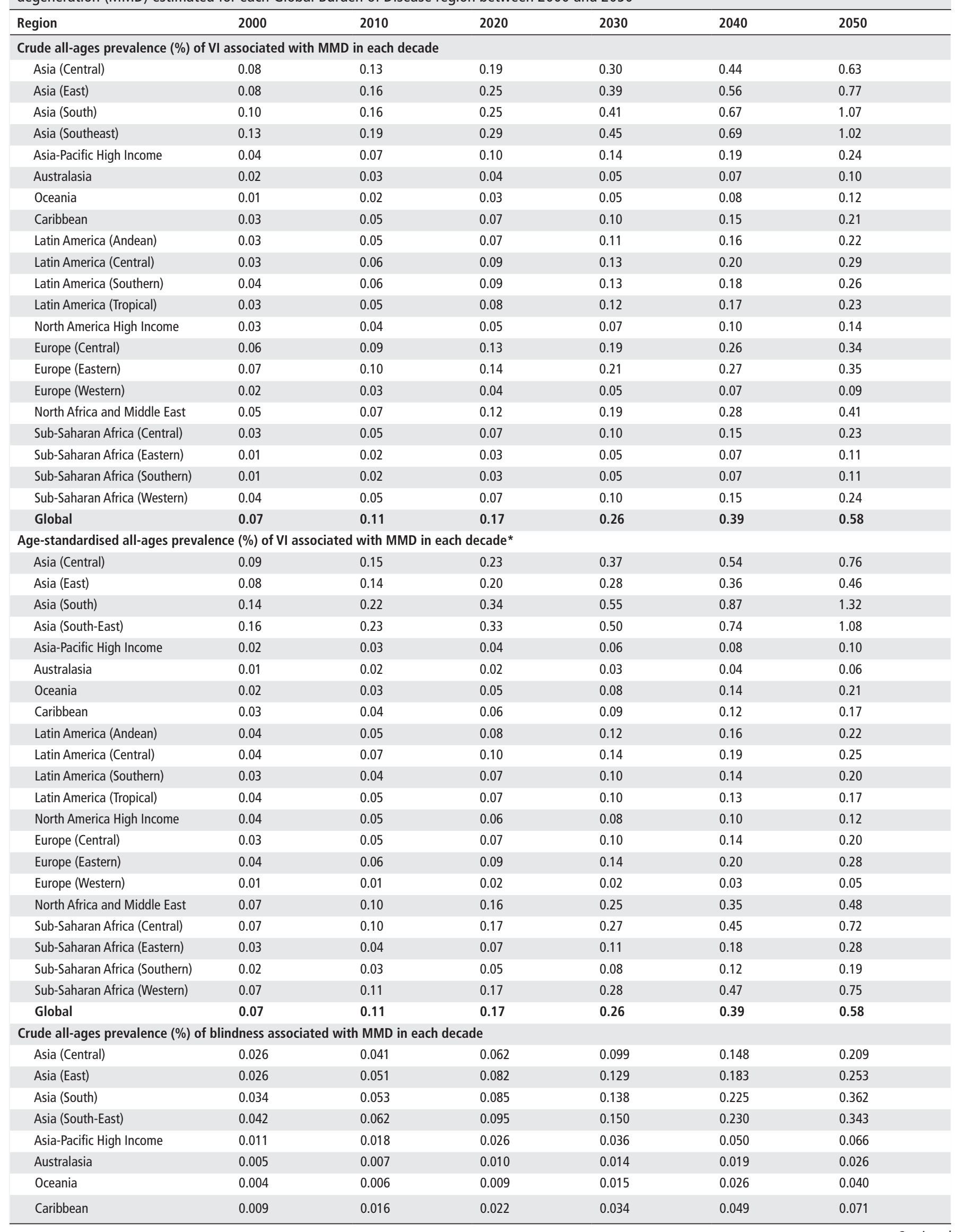

Continued 


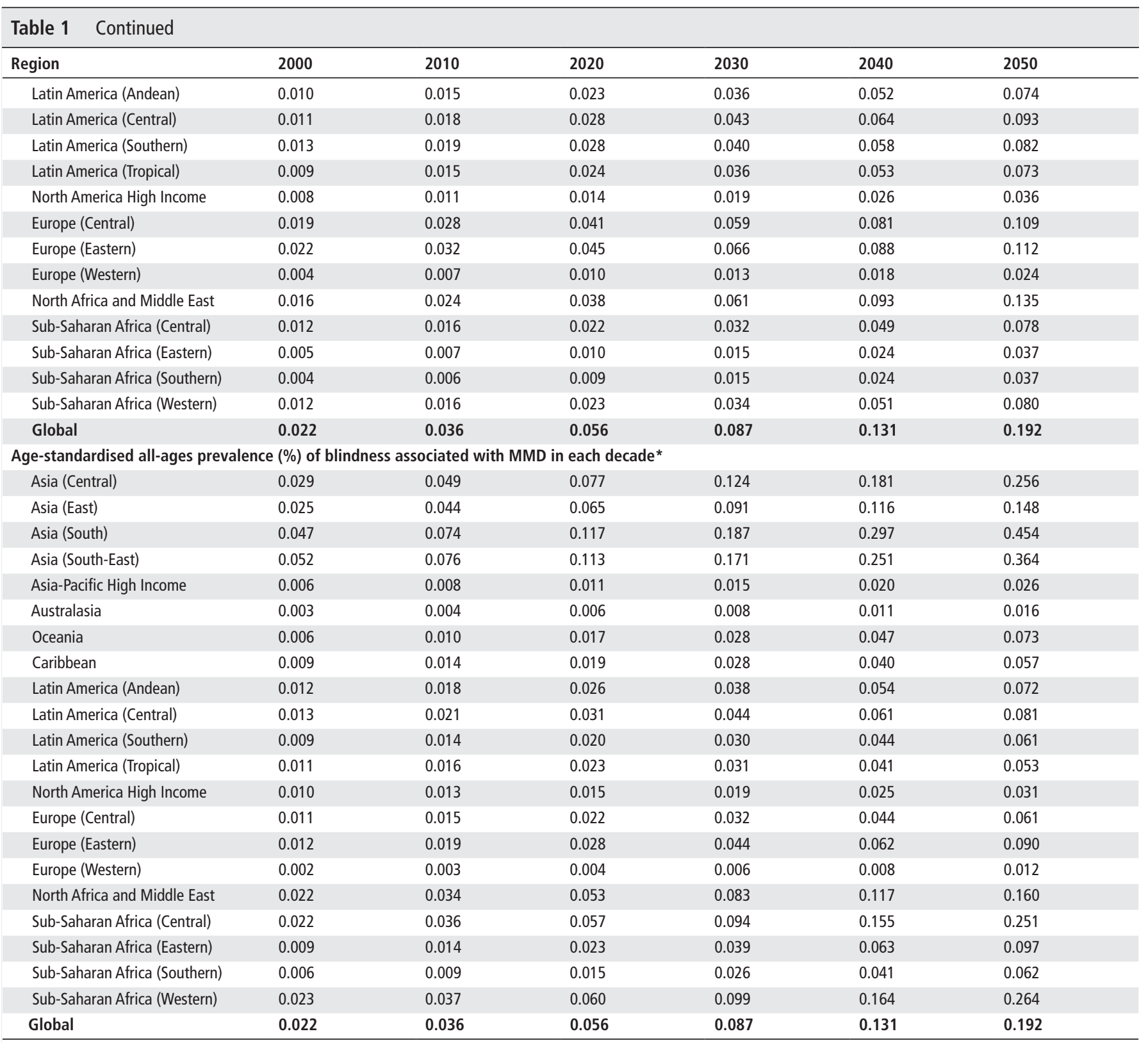

${ }^{*}$ Age standardisation was to the global population, but standardisation may not be robust when event rates are low.

Analysis of variance across available studies shows an association between VI attributed to MMD and a country's HE. The nature of the relationship is not clear from this analysis. Robust methodology in each primary study attributes VI cause directly to $\mathrm{MMD}$, suggesting a protective effect of $\mathrm{HE}$ preventing MMD from progressing to VI. Timely antivascular endothelial growth factor treatment seems the most likely explanation, but this is only known to be useful in a specific complication of MMD, myopic choroidal neovascularisation, ${ }^{27}$ and was not in widespread use at the time of many of the primary studies. Thus, we have taken the conservative approach of assuming only an association. It is possible that a related factor-for example, better refractive care leading to people being categorised into different levels of VI-is responsible for the relationship between HE and MMD vision outcomes. Our model refers to 'VI associated with MMD', and we used this location effect at the country level. It is possible that there are also effects of gender, ethnicity and generation, but there are insufficient data to determine the effects other than the level of myopia and location at this stage.

We predict the prevalence of VI associated with MMD will increase eightfold between 2000 and 2050. This dramatic increase is due to the compounding effects of increasing myopia, shifting high myopia demographics towards older age, increasing mean spherical equivalent (and axial length) and the ageing population. In terms of number rather than prevalence, the increase is predicted to be 13 times for VI associated with MMD. In global terms, the 'myopia epidemic' has been a relatively recent phenomenon, most marked in young people. This means that while there is now a high prevalence of high myopia in young people, the impact of high myopia-associated complications will only become evident over the following decades, as these people age and become susceptible to MMD VI.

We have assumed a constant proportion of people with high myopia having MMD VI between 2000 and 2050. This may be conservative because the predicted rise in average 
spherical equivalent within the high myopia group is likely to increase the risk of $\mathrm{VI},{ }^{245}$ and we have not included the possibility that people with low to moderate myopia can develop MMD VI. ${ }^{28}$ Counteracting these factors, successful myopia prevention or control strategies, together with improved detection and treatment of MMD, could reduce the number of people progressing to VI. Additionally, changes in the myopia causation mix over time may lead to different rates of VI from MMD. On balance, we feel our model is based on the evidence as it exists today, and is a reasonable balance of these factors.

Our study design has some potential limitations. The first is the spread of primary evidence around the world-there could be location and ethnicity effects that have not been detected in this analysis. The primary evidence comes from the Asia Pacific High Income, East Asia, Central Europe and Western Europe regions. No study provided evidence that ethnicity would affect susceptibility to VI associated with MMD in eyes of the same age, size, refractive error and country location, although it is possible that differences do exist. We mitigated against errors induced by the representation of regions in the primary evidence by using MMD VI as a proportion of high myopia, which seems likely to be the strongest control variable between populations when meta-analysing and modelling data. The second limitation is that there was only one available peer-reviewed global model of high myopia prevalence, ${ }^{2}$ which we used on the understanding that limitations in that paper would be transferred to this work. The third limitation is a lack of primary data differentiating MMD VI into small enough age groups. We used the age spread from the six studies with the best age group differentiation. Fourth, projecting based on current information has the potential to miss variations over time, such as improved myopia control or management of MMD. Fifth, while our modelling suggested that country-level HE is associated with the distribution of MMD VI in 2015, we did not include changes in HE in our future projections. It is hoped that access to and quality of healthcare will increase significantly over the next 30 years. If improvements in access to and quality of healthcare enable improved visual outcomes in MMD over time, our model will overestimate the future problem. Sixth, we assume there are no gender or cohort effects on the susceptibility to VI associated with MMD of an eye of the same age, size, refractive error and country location. We view this as a conservative approach since there is no consistent evidence showing gender and cohort effects on MMD VI at this time.

Strategies to prevent myopia, such as increased time outside or different educational approaches, have the potential to reduce the future prevalence of high myopia and the risk of developing MMD VI. Likewise, strategies to reduce myopia progression, such as low-dose atropine and specific spectacle and contact lens designs, have similar potential. Improved surveillance, access to care, case management and treatment options for avoiding VI associated with MMD could also decrease risk over time. ${ }^{28}$

Additional discussion is available in online supplementary file 1 .

In conclusion, our systematic review, meta-analysis and modelling of the evidence describing VI and blindness associated with MMD have significant implications for planning comprehensive eye care services globally, including managing and, if possible, preventing the ocular complications and VI that may occur among almost a billion people with high myopia.

Acknowledgements We acknowledge David A Wilson, formerly of the Brien Holden Vision Institute, Sydney Australia, for his integrity, collegiality, and tireless contributions to improving eye care in general, and to this paper specifically. He sadly passed away before we could complete this paper together.

Contributors TRF: guarantor for the paper and involved in all aspects of its design, conception, analysis, manuscript creation and editing. MJ: involved in manuscript creation and editing. KSN: involved in conception, project design and editing. PS: involved in analysis and editing. TN: involved in project design and data analysis. SMH: involved in data analysis and editing. TYW: involved in conception and editing. SR: involved in design, conception, analysis, manuscript creation and editing.

Funding This work was supported by a public health grant from the Brien Holden Vision Institute, Sydney, Australia.

Competing interests The authors have no relevant proprietary interests. TRF: consultant for Brien Holden Vision Institute. MJ: no financial disclosures. KSN: no financial disclosures. PS: no financial disclosures. TN: no financial disclosures. SMH: no financial disclosures. TYW: consultant and on advisory boards for Allergan, Bayer and Novartis. SR: consultant for Brien Holden Vision Institute and previously worked for Thea Pharmaceuticals (2008-2010).

\section{Patient consent Not required.}

Data sharing statement In addition to the article and the online supplementary file, the authors are willing to share country-specific and/or age-specific data generated by the model designed in this study.

Provenance and peer review Not commissioned; externally peer reviewed.

Author note This submission has not been published anywhere previously and it is not simultaneously being considered for any other publication.

Open access This is an Open Access article distributed in accordance with the Creative Commons Attribution Non Commercial (CC BY-NC 4.0) license, which permits others to distribute, remix, adapt, build upon this work non-commercially, and license their derivative works on different terms, provided the original work is properly cited and the use is non-commercial. See: http://creativecommons.org/ licenses/by-nc/4.0/

(c) Article author(s) (or their employer(s) unless otherwise stated in the text of the article) 2018. All rights reserved. No commercial use is permitted unless otherwise expressly granted.

\section{REFERENCES}

1 Bourne RR, Stevens GA, White RA, et al. Causes of vision loss worldwide, 1990-2010: a systematic analysis. Lancet Glob Health 2013;1:e339-49.

2 Holden BA, Fricke TR, Wilson DA, et al. Global prevalence of myopia and high myopia and temporal trends from 2000 through 2050. Ophthalmol 2016;123:1036-42.

3 Ohno-Matsui K, Lai TYY, Lai C-C, et al. Updates of pathologic myopia. Prog Retin Eye Res 2016:52:156-87

4 Verhoeven VJ, Wong KT, Buitendijk GH, et al. Visual consequences of refractive errors in the general population. Ophthalmology 2015;122:101-9.

5 Tideman JW, Snabel MC, Tedja MS, et al. Association of axial length with risk of uncorrectable visual impairment for Europeans with myopia. JAMA Ophthalmol 2016:134:1355-63.

6 Yamada M, Hiratsuka Y, Roberts CB, et al. Prevalence of visual impairment in the adult Japanese population by cause and severity and future projections. Ophthalmic Epidemiol 2010;17:50-7.

7 Nakamura Y, Tomidokoro A, Sawaguchi S, et al. Prevalence and causes of low vision and blindness in a rural Southwest Island of Japan. Ophthalmology 2010;117:2315-21.

$8 \mathrm{Xu} \mathrm{L}$, Wang Y, Li Y, et al. Causes of blindness and visual impairment in urban and rural areas in Beijing. Ophthalmology 2006;113:1134.e1-1134.e11.

9 Liang YB, Friedman DS, Wong TY, et al. Prevalence and causes of low vision and blindness in a rural Chinese adult population. Ophthalmology 2008;115:1965-72.

10 Tang $Y$, Wang $X$, Wang J, et al. Prevalence and causes of visual impairment in a Chinese adult population. Ophthalmology 2015;122:1480-8.

11 Zhao J, Ellwein LB, Cui $\mathrm{H}$, et al. Prevalence of vision impairment in older adults in rura China: the China Nine-Province Survey. Ophthalmology 2010;117:409-16.

12 Wu L, Sun X, Zhou X, et al. Causes and 3-year-incidence of blindness in Jing-An District, Shanghai, China 2001-2009. BMC Ophthalmol 2011;11:1-6.

13 Huang S, Zheng Y, Foster PJ, et al. Prevalence and causes of visual impairment in Chinese adults in urban Southern China. Arch Ophthalmol 2009:127:1362-7.

14 Tsai IL, Woung LC, Tsai CY, et al. Trends in blind and low vision registrations in Taipei City. Eur J Ophthalmol 2008;18:118-24.

15 Liu JH, Cheng CY, Chen SJ, et al. Visual impairment in a Taiwanese population: prevalence, causes, and socioeconomic factors. Ophthalmic Epidemiol 2001;8:339-50.

16 Hsu W, Cheng C, Liu J, et al. Prevalence and causes of visual impairment in an elderly Chinese population in Taiwan: The Shihpai Eye Study. Ophthalmol 2004;111:62-9.

17 Klaver CC, Wolfs RC, Vingerling JR, et al. Age-specific prevalence and causes of blindness and visual impairment in an older population: the Rotterdam Study. Arch Ophthalmol 1998;116:653-8. 
18 Farber MD. National Registry for the Blind in Israel: estimation of prevalence and incidence rates and causes of blindness. Ophthalmic Epidemiol 2003;10:267-77.

19 Evans JR, Fletcher AE, Wormald RP. MRC trial of assessment \& management of older people in the community. causes of visual impairment in people aged 75 years and older in Britain: an add-on study to the MRC Trial of Assessment and Management of Older People in the Community. Br J Ophthalmol 2004;88:365-70.

20 Buch H, Vinding T, Nielsen NV. Prevalence and causes of visual impairment according to World Health Organization and United States criteria in an aged, urban Scandinavian population The Copenhagen City Eye Study. Ophthalmology 2001; 108:2347-57

21 Nowak MS, Smigielski J. The Prevalence of Age-Related Eye Diseases and Cataract Surgery among Older Adults in the City of Lodz, Poland. J Ophthalmol 2015;2015:1-7.

22 United Nations Population Division, Department of Economic and Social Affairs. World Population Prospects: the 2015 Revision. United Nations. 2015. https://esa.un. org/unpd/wpp/ (accessed 19 Feb 2016).
23 World Health Organization, Brien Holden Vision Institute. The impact of myopia and high myopia. Report of the Joint World Health Organization - Brien Holden Vision Institute (WHO-BHVI) Global Scientific Meeting on Myopia held at the University of New South Wales in Sydney, Australia, 16-18 March 2015. Geneva: World Health Organization. 2016.

24 Spaide RF, Ohno-Matsui K, Yannuzzi LA, et al. Pathologic Myopia. New York: Springer, 2014.

25 World Health Organization. International Classification of Disease (ICD)-10. 2016. http://apps.who.int/classifications/icd10/browse/2016/en (accessed 20 Dec 2016).

26 World Bank. World Development Indicators. 2018. http://data.worldbank.org/ products/wdi (accessed 23 Mar 2017).

27 Cheung CMG, Arnold JJ, Holz FG, et al. Myopic Choroidal Neovascularization. Ophthalmology 2017;124:1690-

28 Wong TY, Ferreira A, Hughes $R$, et al. Epidemiology and disease burden of pathologic myopia and myopic choroidal neovascularization: an evidence-based systematic review. Am J Ophthalmol 2014;157:9-25. 